\title{
Supporting Information (SI): Comparing modeled and measured mercury speciation in contaminated groundwater: Importance of dissolved organic matter composition
}

Jan-Helge Richard*, Cornelia Bischoff, Harald Biester

Institut für Geoökologie, AG Umweltgeochemie, Technische Universität Braunschweig, Germany

\section{9 pages}

Figure S1. Scheme of analytical protocol followed for $\mathrm{Hg}$ speciation measurements.

Figure S2. Inorganic Hg-speciation from all sites calculated by the investigated codes subdivided for the applied codes.

Figure S3. Inorganic $\mathrm{Hg}$-speciation from all sites calculated by the investigated codes subdivided for the predicted $\mathrm{Hg}$-species.

Figure S4. Comparison of measured and predicted concentrations of dissolved elemental mercury $(\operatorname{Hg}(0))$.

Equation S1. Calculation of root-mean-square errors (RMSEs).

Equation S2. Calculation of mean signed differences (MSDs).

Table S1. Chemical composition of groundwater samples from Site A.

Table S2. Chemical composition of groundwater samples from Site B.

Table S3. Chemical composition of groundwater samples from Site C.

Table S4. Used equilibrium constants (log K values) of aqueous $\mathrm{Hg}$ species and complexes.

Table S5. Analytical details of $\mathrm{Hg}$ measurements.

Table S6. Limits of quantification, standard reference materials, and recovery rates for different analytes.

Table S7. RMSEs and MSDs of modeled vs. measured $\mathrm{Hg}$ speciation.

Table S8. Linear correlations between measured and modeled total inorganic $\mathrm{Hg}$ concentrations $(\log (\mathrm{M}))$ in groundwater.

Table S9. Linear correlations between measured and modeled DOM bound $\mathrm{Hg}$ concentrations $(\log (\mathrm{M}))$ in groundwater. 


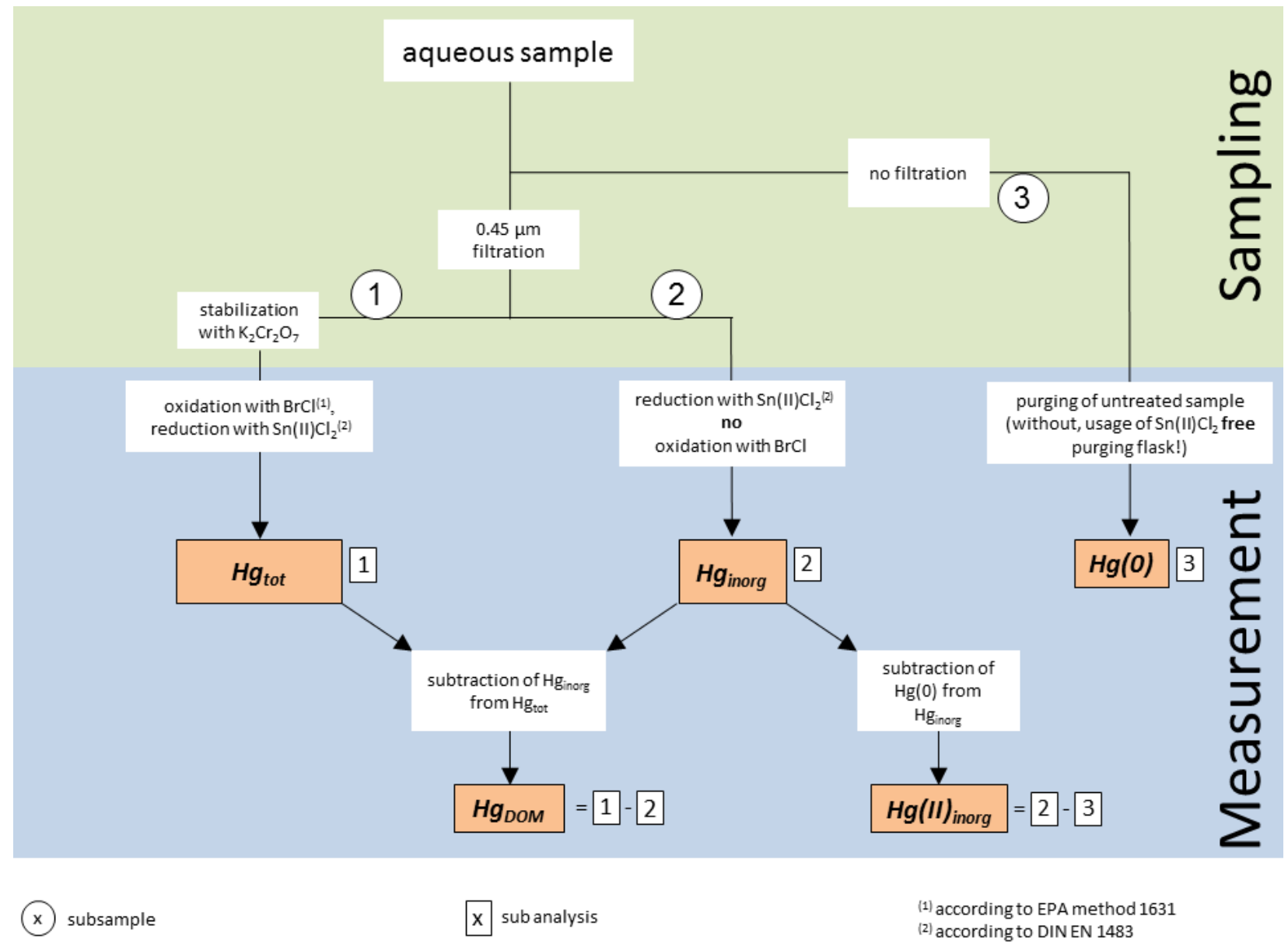

Figure S1. Scheme of the analytical protocol followed for the Hg speciation measurements. 


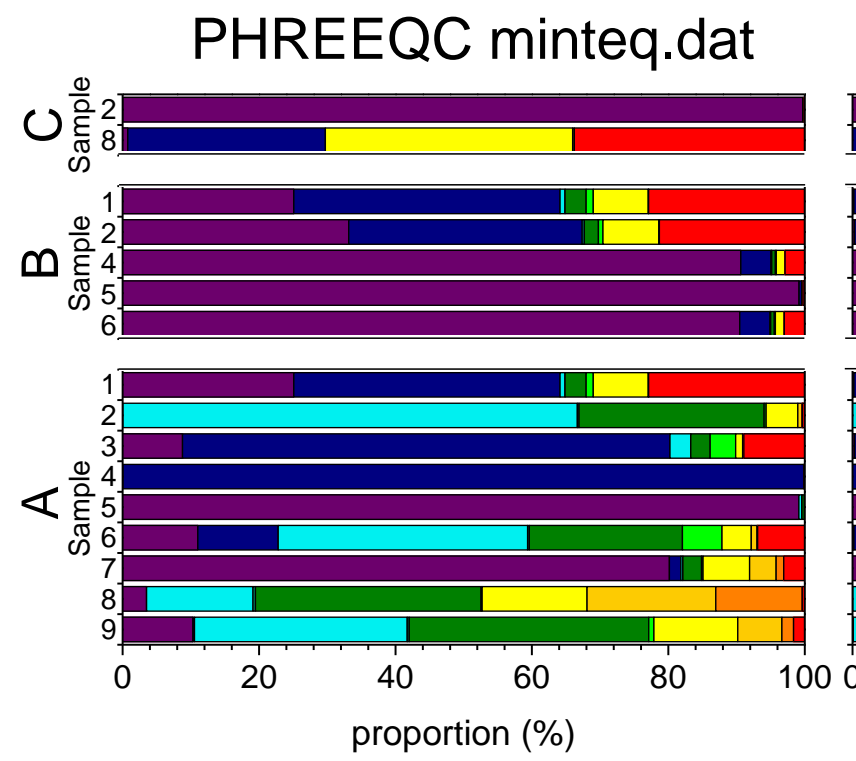

\section{PHREEQC minteq.v4.dat}
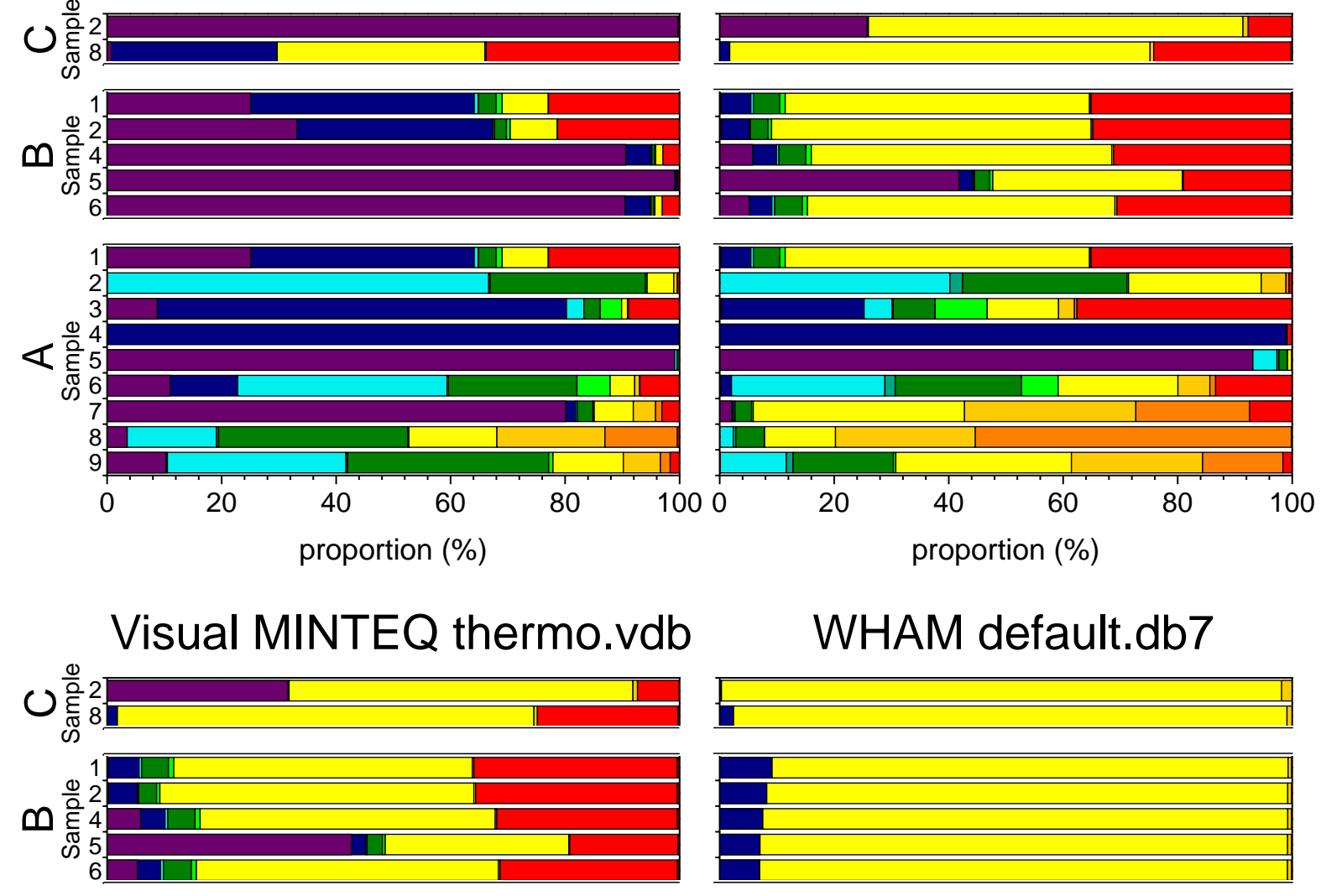

\section{WHAM default.db7}
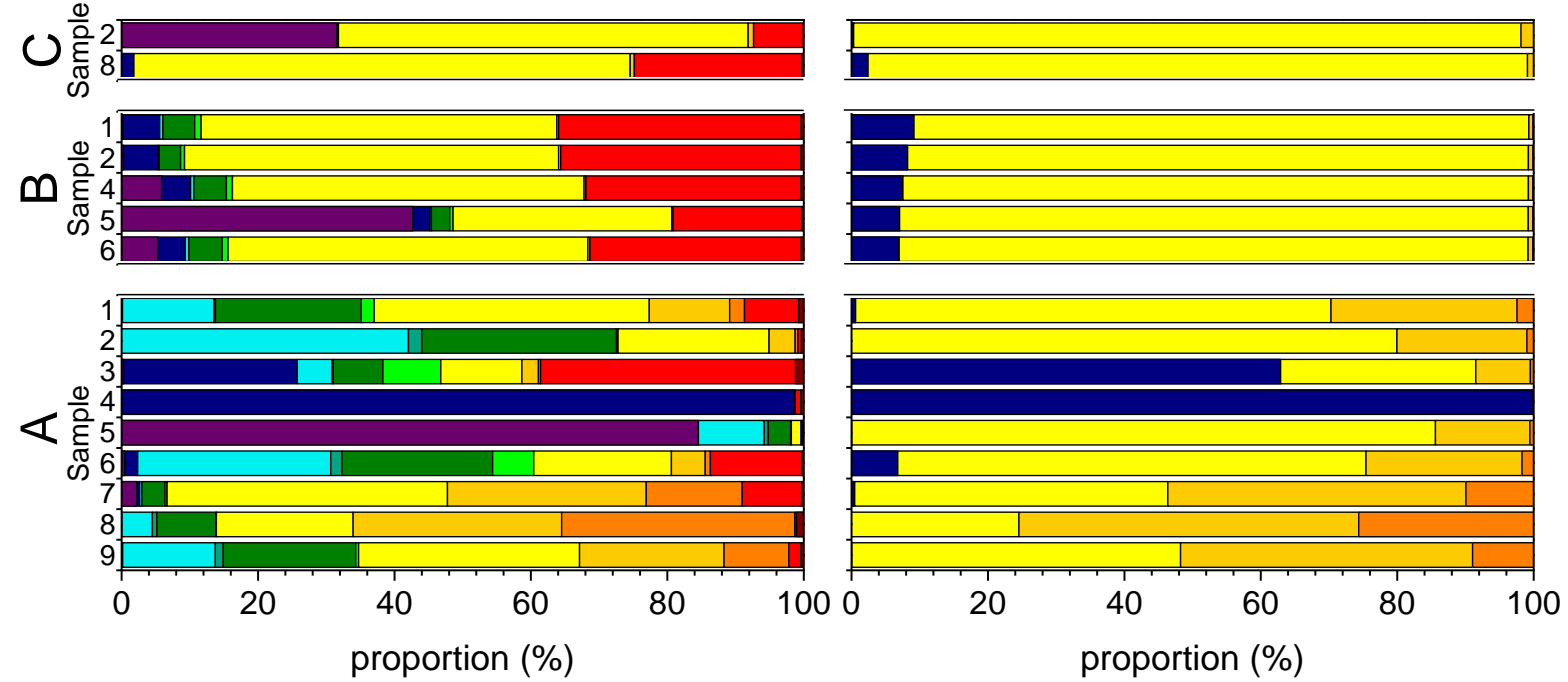

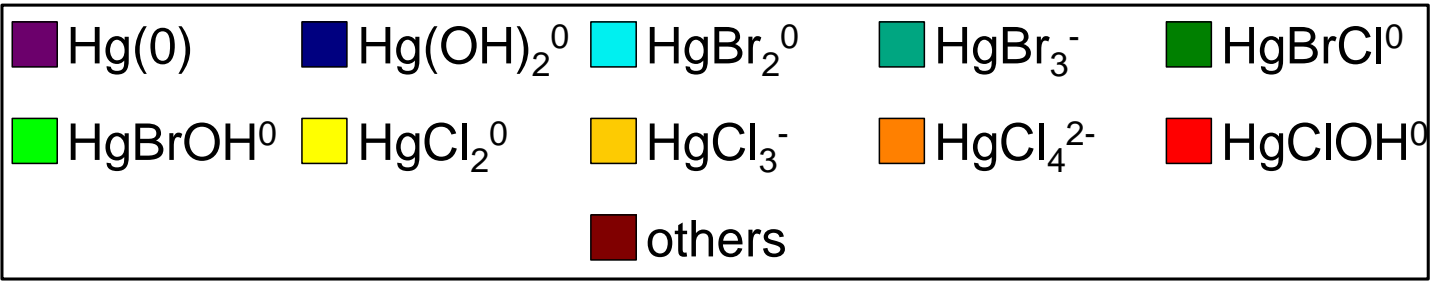

Figure S2. Relative proportions of inorganic species as calculated for samples from all sites, subdivided for the applied database. Only the samples that were modeled in scenario 1 are shown. 


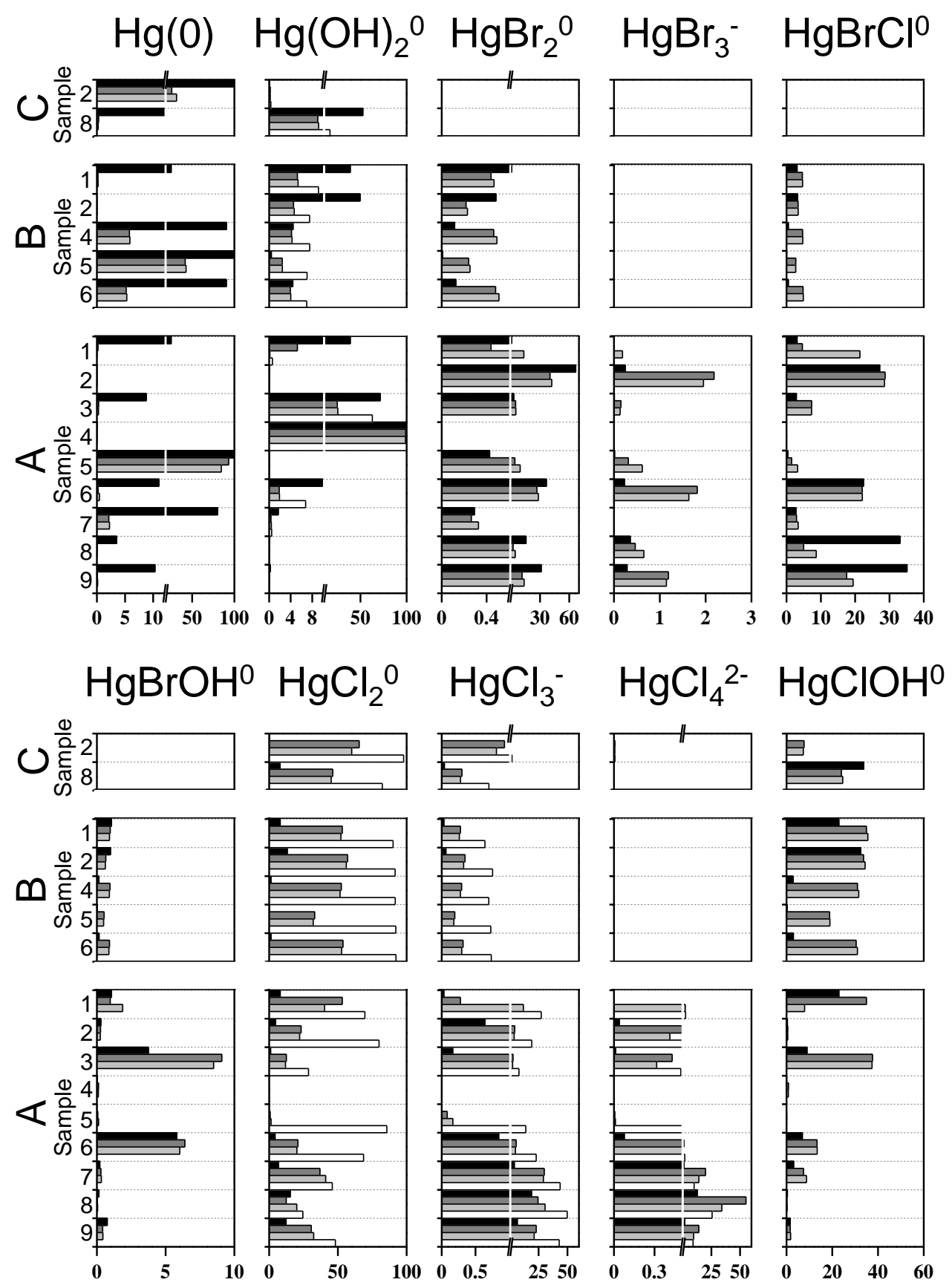

PHREEQC minteq.dat $\square$ PHREEQC minteq.v4.dat

Visual MINTEQ thermo.vdb $\square$ WHAM default.db7

Figure S3. Relative proportions of inorganic species as calculated for samples from all sites, subdivided for the ten most abundant $\mathrm{Hg}$ complexes. Only the samples that were modeled in scenario 1 are shown. 

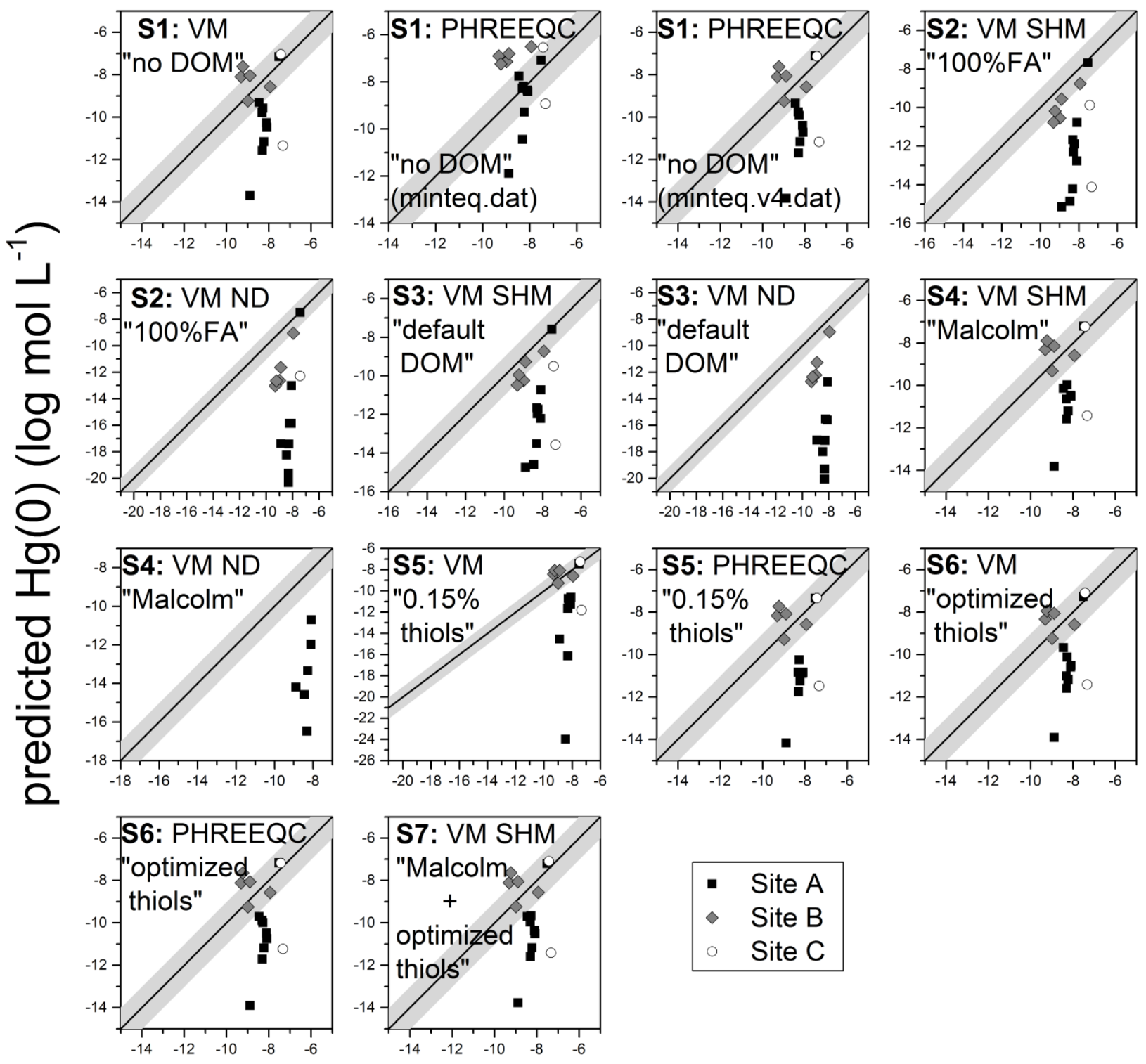

$$
\begin{array}{ll}
- & \text { Site A } \\
- & \text { Site B } \\
- & \text { Site C }
\end{array}
$$

\section{measured $\mathrm{Hg}(0)\left(\log \mathrm{mol} \mathrm{L}^{-1}\right)$}

Figure S4. Comparison of measured and predicted concentrations of dissolved elemental mercury $(\mathrm{Hg}(0))$. "S" denotes the scenario and VM refers to Visual MINTEQ. Note the different scales for each scenario. The solid line and shaded area represent the 1:1 line and one order of magnitude alongside the 1:1 line, respectively. 
Equation S1. Calculation of root-mean-square errors (RMSEs).

$R M S E=\sqrt{\frac{\sum\left(x_{\text {predicted }}-x_{\text {measured }}\right)^{2}}{n}}$

with:

$\mathbf{X}_{\text {predicted }}$

$\mathrm{X}_{\text {measured }}$

n model predicted $\mathrm{Hg}$ concentration of respective $\mathrm{Hg}$ species $\left(\log \mathrm{mol} \mathrm{L}^{-1}\right)$ measured $\mathrm{Hg}$ concentration of respective $\mathrm{Hg}$ species $\left(\log \mathrm{mol} \mathrm{L}^{-1}\right)$ total number of samples

Equation S2. Calculation of mean signed differences (MSDs).

$M S D=\frac{\sum\left(x_{\text {predicted }}-x_{\text {measured }}\right)}{n}$

with:

$\mathbf{X}_{\text {predicted }}$

$\mathrm{X}_{\text {measured }}$

n model predicted $\mathrm{Hg}$ concentration of respective $\mathrm{Hg}$ species $\left(\log \mathrm{mol} \mathrm{L}^{-1}\right)$ measured $\mathrm{Hg}$ concentration of respective $\mathrm{Hg}$ species $\left(\log \mathrm{mol} \mathrm{L}^{-1}\right)$ total number of samples 
Table S1. Hydrochemical composition of groundwater samples from Site A.

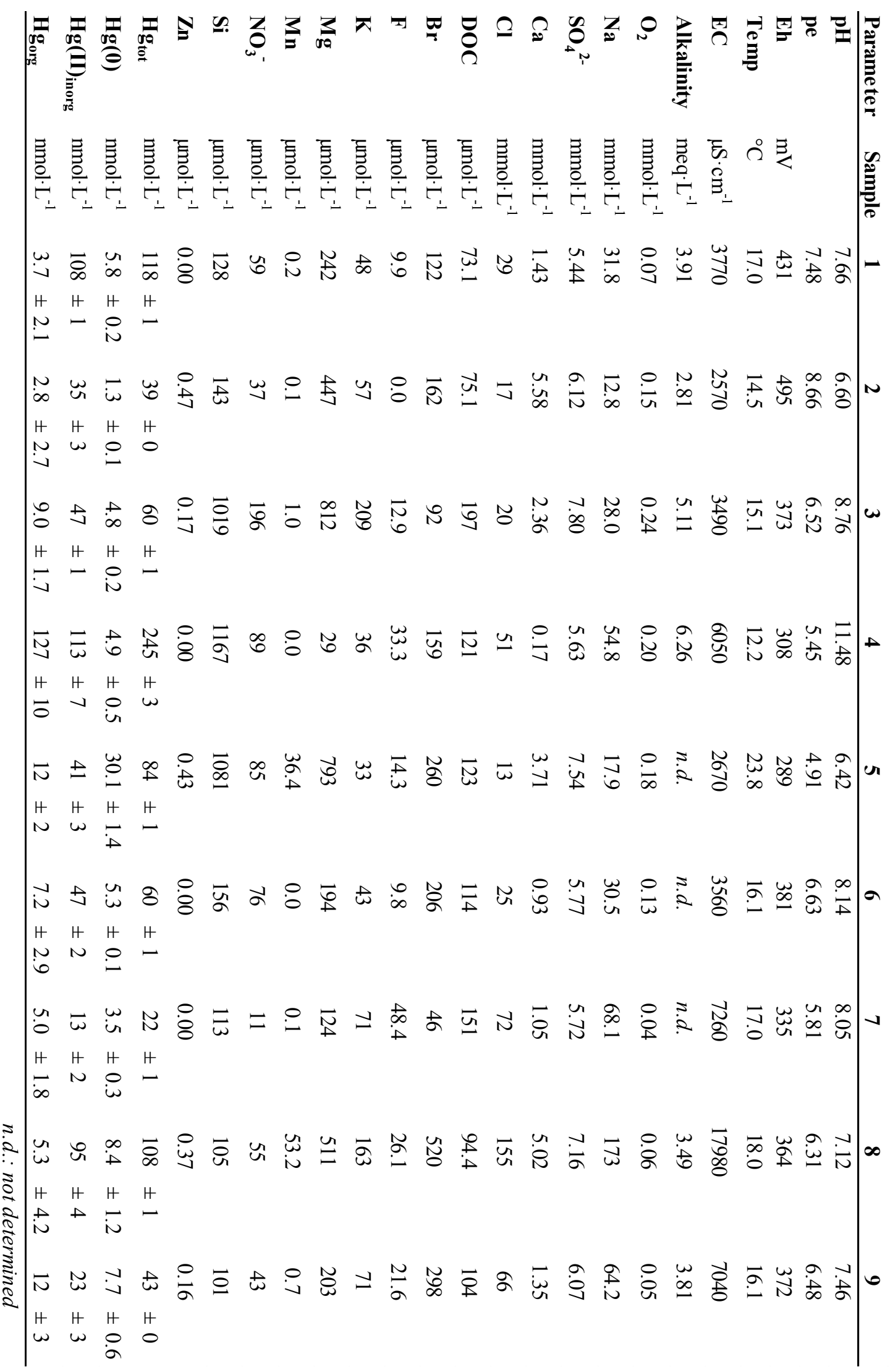


Table S2. Hydrochemical composition of groundwater samples from Site B.

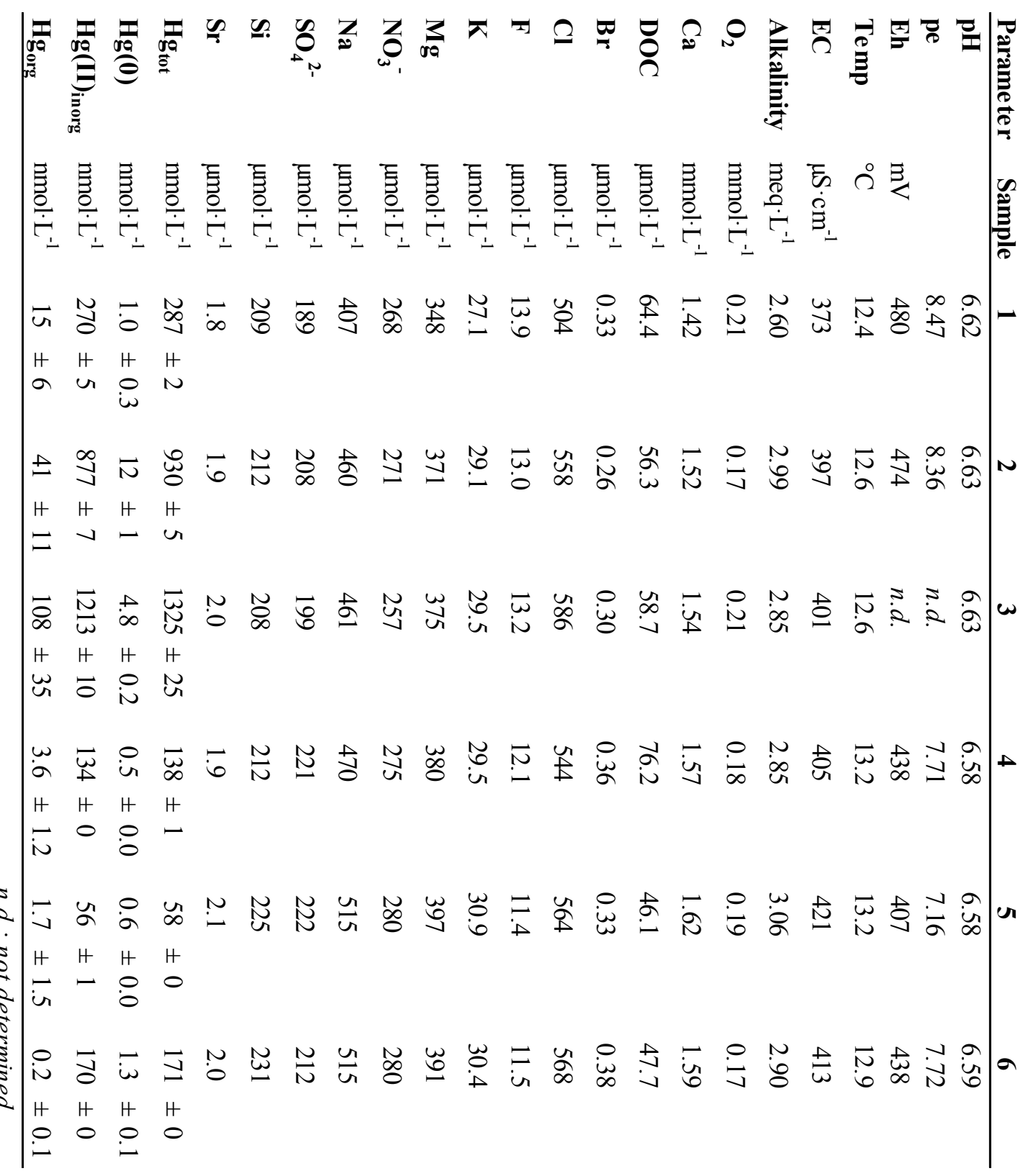


Table S3. Hydrochemical composition of groundwater samples from Site C.

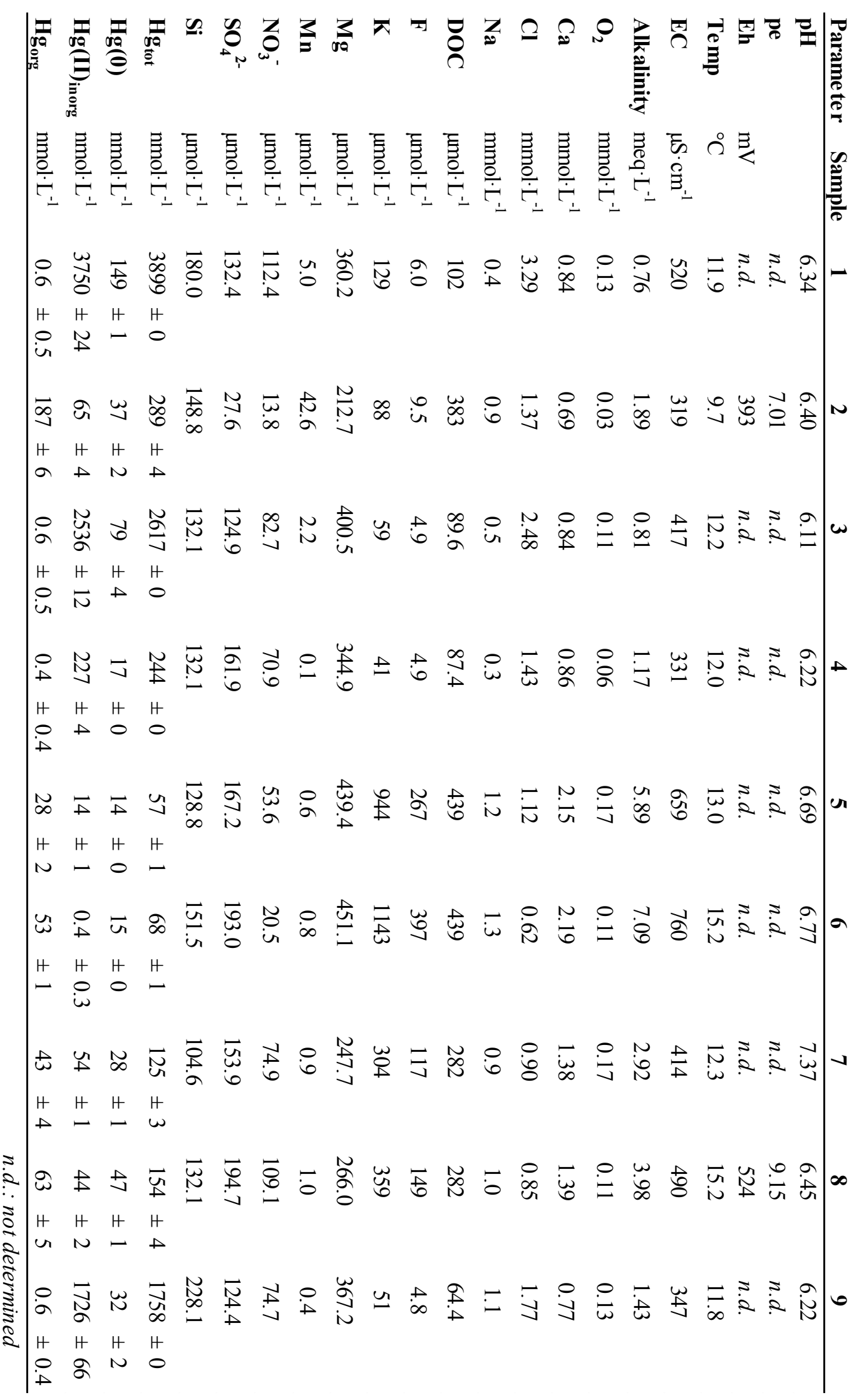


Table S4. Used equilibrium constants (log $\mathrm{K}$ values) of aqueous $\mathrm{Hg}$ species and complexes.

AQUEOUS COMPLEXES

\begin{tabular}{|c|c|c|c|c|c|c|c|}
\hline \multirow[t]{2}{*}{ Name } & \multirow[t]{2}{*}{ Reaction } & \multirow{2}{*}{$\begin{array}{c}\text { PHREEQC } \\
\text { minteq.dat } \\
\log K\end{array}$} & \multirow{2}{*}{$\begin{array}{c}\text { PHREEQC } \\
\text { minteq.v4.dat } \\
\log \mathrm{K}\end{array}$} & \multicolumn{2}{|c|}{$\begin{array}{l}\text { Vis ual Minteq the rmo.vdb } \\
\text { and shmgeneric } 14 . v d b\end{array}$} & \multicolumn{2}{|c|}{$\begin{array}{c}\text { WHAM } \\
\text { default.db7 }\end{array}$} \\
\hline & & & & $\log K$ & $\Delta \mathbf{L K}_{2}$ & $\log K$ & $\Delta \mathbf{L K}_{2}$ \\
\hline $\mathrm{Hg}\left(\mathrm{CO}_{3}\right)_{2}{ }^{2-}$ & $\begin{array}{l}\mathrm{Hg}(\mathrm{OH}) 2+2 \mathrm{H}++ \\
2 \mathrm{CO} 3-2=\mathrm{Hg}(\mathrm{CO} 3) 2-2 \\
+2 \mathrm{H} 2 \mathrm{O}\end{array}$ & & 21.772 & 21.74 & & & \\
\hline $\operatorname{Hg}(\mathrm{HS})_{2}$ & $\begin{array}{l}2 \mathrm{H}++2 \mathrm{HS}-+\mathrm{Hg}(\mathrm{OH}) 2 \\
=\mathrm{Hg}(\mathrm{HS}) 2+2 \mathrm{H} 2 \mathrm{O}\end{array}$ & 43.8178 & $44.516 / 45.242 *$ & $44.58 / 45.242 *$ & & & \\
\hline $\operatorname{Hg}\left(\mathrm{N}_{3}\right)_{2}$ & $\begin{array}{l}\mathrm{Hg}(\mathrm{OH}) 2+2 \mathrm{~N} 3-1+2 \\
\mathrm{H}^{+}=\mathrm{Hg}(\mathrm{N} 3) 2+2 \mathrm{H}^{+}\end{array}$ & & & 21.52 & & & \\
\hline $\mathrm{Hg}\left(\mathrm{NH}_{3}\right)_{2}{ }^{2+}$ & $\begin{array}{l}\mathrm{Hg}(\mathrm{OH}) 2+2 \mathrm{NH} 4+= \\
\mathrm{Hg}(\mathrm{NH} 3) 2+2+2 \mathrm{H} 2 \mathrm{O}\end{array}$ & 5.0341 & 5.506 & 5.47 & & & \\
\hline $\mathrm{Hg}\left(\mathrm{NH}_{3}\right)_{3}{ }^{2+}$ & $\begin{array}{l}\mathrm{Hg}(\mathrm{OH}) 2+3 \mathrm{NH} 4+= \\
\mathrm{Hg}(\mathrm{NH} 3) 3+2+\mathrm{H}++ \\
2 \mathrm{H} 2 \mathrm{O}\end{array}$ & -3.2493 & -3.138 & & & & \\
\hline $\mathrm{Hg}\left(\mathrm{NH}_{3}\right)_{4}{ }^{2+}$ & $\begin{array}{l}\mathrm{Hg}(\mathrm{OH}) 2+4 \mathrm{NH} 4+= \\
\mathrm{Hg}(\mathrm{NH} 3) 4+2+2 \mathrm{H}++ \\
2 \mathrm{H} 2 \mathrm{O}\end{array}$ & -11.7307 & -11.482 & -11.52 & & & \\
\hline $\mathrm{Hg}\left(\mathrm{NO}_{2}\right)_{2}$ & $\begin{array}{l}\mathrm{Hg}(\mathrm{OH}) 2+2 \mathrm{NO} 2-1+2 \\
\mathrm{H}+=\mathrm{Hg}(\mathrm{NO} 2) 2+2 \\
\mathrm{H} 2 \mathrm{O}\end{array}$ & & & 16.07 & & & \\
\hline $\mathrm{Hg}\left(\mathrm{NO}_{2}\right)_{3}{ }^{-}$ & $\begin{array}{l}\mathrm{Hg}(\mathrm{OH}) 2+3 \mathrm{NO} 2-1+2 \\
\mathrm{H}+=\mathrm{Hg}(\mathrm{NO} 2) 3-+2 \\
\mathrm{H} 2 \mathrm{O}\end{array}$ & & & 17.61 & & & \\
\hline $\mathrm{Hg}\left(\mathrm{NO}_{2}\right)_{4}{ }^{2-}$ & $\begin{array}{l}\mathrm{Hg}(\mathrm{OH}) 2+4 \mathrm{NO} 2-1+2 \\
\mathrm{H}+=\mathrm{Hg}(\mathrm{NO} 2) 4-2+2 \\
\mathrm{H} 2 \mathrm{O}\end{array}$ & & & 18.02 & & & \\
\hline $\mathrm{Hg}\left(\mathrm{NO}_{3}\right)_{2}$ & $\begin{array}{l}2 \mathrm{H}++\mathrm{Hg}(\mathrm{OH}) 2+ \\
2 \mathrm{NO} 3-=\mathrm{Hg}(\mathrm{NO} 3) 2+ \\
2 \mathrm{H} 2 \mathrm{O}\end{array}$ & 4.7791 & 5.38 & 5.38 & & & \\
\hline $\mathrm{Hg}(\mathrm{OH})_{3}^{-}$ & $\begin{array}{l}\mathrm{H} 2 \mathrm{O}+\mathrm{Hg}(\mathrm{OH}) 2= \\
\mathrm{Hg}(\mathrm{OH}) 3-+\mathrm{H}+\end{array}$ & -15.0042 & -14.897 & & & & \\
\hline $\mathrm{Hg}(\mathrm{RO})_{2}$ & $\begin{array}{l}2 \mathrm{ROH}+\mathrm{Hg}(\mathrm{OH}) 2= \\
\mathrm{Hg}(\mathrm{RO}) 2+2 \mathrm{H}+\end{array}$ & & & (6.7) & 3.1 & & \\
\hline $\mathrm{Hg}\left(\mathrm{SO}_{3}\right)_{2}{ }^{2-}$ & $\begin{array}{l}\mathrm{Hg}(\mathrm{OH}) 2+2 \mathrm{SO} 3-2+ \\
2 \mathrm{H}+=\mathrm{Hg}(\mathrm{SO} 3) 2-2+ \\
\mathrm{H} 2 \mathrm{O}\end{array}$ & & & 29.62 & & & \\
\hline $\mathrm{Hg}\left(\mathrm{SO}_{3}\right)_{2}{ }^{4-}$ & $\begin{array}{l}\mathrm{Hg}(\mathrm{OH}) 2+3 \mathrm{SO} 3-2+ \\
2 \mathrm{H}+=\mathrm{Hg}(\mathrm{SO} 3) 2-4+ \\
\mathrm{H} 2 \mathrm{O}\end{array}$ & & & 30.66 & & & \\
\hline
\end{tabular}


Table S4. Used equilibrium constants (log K values) of aqueous $\mathrm{Hg}$ species and complexes - Continued.

AQUEOUS COMPLEXES

\begin{tabular}{|c|c|c|c|c|c|c|c|}
\hline \multirow[t]{2}{*}{ Name } & \multirow[t]{2}{*}{ Reaction } & \multirow{2}{*}{$\begin{array}{c}\text { PHREEQC } \\
\text { minteq.dat } \\
\qquad \log K\end{array}$} & \multirow{2}{*}{$\begin{array}{c}\text { PHREEQC } \\
\text { minteq.v4.dat } \\
\log \mathrm{K}\end{array}$} & \multicolumn{2}{|c|}{$\begin{array}{l}\text { Vis ual Minteq the rmo.vdb } \\
\text { and shmgeneric } 14 . v d b\end{array}$} & \multicolumn{2}{|c|}{$\begin{array}{c}\text { WHAM } \\
\text { default.db7 }\end{array}$} \\
\hline & & & & $\log K$ & $\Delta \mathbf{L K}_{2}$ & $\log K$ & $\Delta \mathbf{L K}_{2}$ \\
\hline $\mathrm{Hg}\left(\mathrm{SO}_{4}\right)_{2}{ }^{2-}$ & $\begin{array}{l}\mathrm{Hg}(\mathrm{OH}) 2+3 \mathrm{SO} 4-2+ \\
2 \mathrm{H}+=\mathrm{Hg}(\mathrm{SO} 4) 2-2+2 \\
\mathrm{H} 2 \mathrm{O}\end{array}$ & & & 9.64 & & & \\
\hline $\mathrm{Hg}(\mathrm{SR})_{2}$ & $\begin{array}{l}2 \mathrm{RS}-+\mathrm{Hg}(\mathrm{OH}) 2+2 \mathrm{H}+ \\
=\mathrm{Hg}(\mathrm{SR}) 2+2 \mathrm{H} 2 \mathrm{O}\end{array}$ & & $48.142^{\circ}$ & $48.142^{\circ}$ & & & \\
\hline $\mathrm{Hg}(\mathrm{SR})_{2}$ & $\mathrm{Hg} 2++2 \mathrm{RS}-=\mathrm{Hg}(\mathrm{SR}) 2$ & & & & & $42^{\circ}$ & \\
\hline $\mathrm{Hg}^{2+}$ & $\begin{array}{l}2 \mathrm{H}++\mathrm{Hg}(\mathrm{OH}) 2=\mathrm{Hg}+2 \\
+2 \mathrm{H} 2 \mathrm{O}\end{array}$ & 6.097 & 6.194 & 6.164 & & & \\
\hline $\mathrm{Hg}_{(\mathrm{aq})}^{\mathbf{0}}$ & $0.5 \mathrm{Hg} 2+2+\mathrm{e}-=\mathrm{Hg}$ & 6.9316 & 6.5667 & 6.5667 & & & \\
\hline $\mathrm{Hg}_{2}{ }^{2+}$ & $\begin{array}{l}4 \mathrm{H}++2 \mathrm{Hg}(\mathrm{OH}) 2+2 \mathrm{e}- \\
=\mathrm{Hg} 2+2+4 \mathrm{H} 2 \mathrm{O}\end{array}$ & 42.987 & 43.185 & & & & \\
\hline $\mathrm{Hg}_{2} \mathrm{OH}^{3+}$ & $\begin{array}{l}2 \mathrm{Hg}(\mathrm{OH}) 2+3 \mathrm{H}+= \\
\mathrm{Hg} 2 \mathrm{OH}+3 \mathrm{H} 2 \mathrm{O}\end{array}$ & & & 9.031 & & & \\
\hline $\mathrm{Hg}_{3}(\mathrm{OH})_{3}{ }^{3+}$ & $\begin{array}{l}3 \mathrm{Hg}(\mathrm{OH}) 2+3 \mathrm{H}^{+}= \\
\mathrm{Hg} 3(\mathrm{OH}) 3+3+3 \mathrm{H} 2 \mathrm{O}\end{array}$ & & & 12.101 & & & \\
\hline $\mathrm{HgBr}^{+}$ & $\begin{array}{l}\mathrm{Br}-+2 \mathrm{H}++\mathrm{Hg}(\mathrm{OH}) 2= \\
\mathrm{HgBr}++2 \mathrm{H} 2 \mathrm{O}\end{array}$ & 15.8347 & 15.803 & 15.77 & & & \\
\hline $\mathrm{HgBr}_{2}$ & $\begin{array}{l}2 \mathrm{Br}-+2 \mathrm{H}++\mathrm{Hg}(\mathrm{OH}) 2 \\
=\mathrm{HgBr} 2+2 \mathrm{H} 2 \mathrm{O}\end{array}$ & 23.6065 & 24.2725 & 24.29 & & & \\
\hline $\mathrm{HgBr}_{3}^{-}$ & $\begin{array}{l}3 \mathrm{Br}-+2 \mathrm{H}++\mathrm{Hg}(\mathrm{OH}) 2 \\
=\mathrm{HgBr} 3-+2 \mathrm{H} 2 \mathrm{O}\end{array}$ & 25.7857 & 26.7025 & 26.67 & & & \\
\hline $\mathrm{HgBr}_{4}^{2-}$ & $\begin{array}{l}4 \mathrm{Br}-+2 \mathrm{H}++\mathrm{Hg}(\mathrm{OH}) 2 \\
=\mathrm{HgBr} 4-2+2 \mathrm{H} 2 \mathrm{O}\end{array}$ & 27.0633 & 27.933 & 27.9 & & & \\
\hline $\mathrm{HgBrCl}$ & $\begin{array}{l}\mathrm{Br}-+\mathrm{Cl}-+2 \mathrm{H}++ \\
\mathrm{Hg}(\mathrm{OH}) 2=\mathrm{HgBrCl}+ \\
2 \mathrm{H} 2 \mathrm{O}\end{array}$ & 22.0145 & 22.1811 & 22.1811 & & & \\
\hline $\mathrm{HgBrOH}$ & $\begin{array}{l}\mathrm{Br}-+\mathrm{H}++\mathrm{Hg}(\mathrm{OH}) 2= \\
\mathrm{HgBrOH}+\mathrm{H} 2 \mathrm{O}\end{array}$ & 11.598 & 12.433 & 12.4 & & & \\
\hline
\end{tabular}


Table S4. Used equilibrium constants (log K values) of aqueous $\mathrm{Hg}$ species and complexes - Continued.

AQUEOUS COMPLEXES

\begin{tabular}{|c|c|c|c|c|c|c|c|}
\hline \multirow[t]{2}{*}{ Name } & \multirow[t]{2}{*}{ Reaction } & \multirow{2}{*}{$\begin{array}{r}\text { PHREEQC } \\
\text { minteq.dat } \\
\log K \\
\end{array}$} & \multirow{2}{*}{$\begin{array}{c}\text { PHREEQC } \\
\text { minteq.v4.dat } \\
\log \mathrm{K} \\
\end{array}$} & \multicolumn{2}{|c|}{$\begin{array}{l}\text { Visual Minteq the rmo.vdb } \\
\text { and shmgene ric 14.vdb }\end{array}$} & \multicolumn{2}{|c|}{$\begin{array}{c}\text { WHAM } \\
\text { de fault.db7 }\end{array}$} \\
\hline & & & & $\log K$ & $\Delta \mathbf{L K}_{\mathbf{2}}$ & $\log K$ & $\Delta \mathbf{L K}_{2}$ \\
\hline $\mathrm{HgCl}^{+}$ & $\begin{array}{l}\mathrm{Cl}-+2 \mathrm{H}++\mathrm{Hg}(\mathrm{OH}) 2= \\
\mathrm{HgCl}++2 \mathrm{H} 2 \mathrm{O}\end{array}$ & 12.85 & 13.494 & 13.49 & & & \\
\hline $\mathrm{HgCl}^{+}$ & $\mathrm{Hg} 2+\mathrm{Cl}-=\mathrm{HgCl}+$ & & & & & 7.21 & \\
\hline $\mathrm{HgCl}_{2}$ & $\begin{array}{l}2 \mathrm{Cl}-+2 \mathrm{H}++\mathrm{Hg}(\mathrm{OH}) 2 \\
=\mathrm{HgCl} 2+2 \mathrm{H} 2 \mathrm{O}\end{array}$ & 19.2203 & 20.194 & 20.19 & & & \\
\hline $\mathrm{HgCl}_{2}$ & $\mathrm{Hg} 2+2 \mathrm{Cl}-=\mathrm{HgCl} 2$ & & & & & 13.98 & \\
\hline $\mathrm{HgCl}_{3}^{-}$ & $\begin{array}{l}3 \mathrm{Cl}-2 \mathrm{H}++\mathrm{Hg}(\mathrm{OH}) 2 \\
=\mathrm{HgCl}-+2 \mathrm{H} 2 \mathrm{O}\end{array}$ & 20.1226 & 21.194 & 21.19 & & & \\
\hline $\mathrm{HgCl}_{3}^{-}$ & $\mathrm{Hg} 2+3 \mathrm{Cl}-=\mathrm{HgCl} 3-$ & & & & & 15.06 & \\
\hline $\mathrm{HgCl}_{4}{ }^{2-}$ & $\mathrm{Hg} 2+4 \mathrm{Cl}-=\mathrm{HgCl} 4-2$ & & & & & 15.42 & \\
\hline $\mathrm{HgCl}_{4}{ }^{2-}$ & $\begin{array}{l}4 \mathrm{Cl}-+2 \mathrm{H}++\mathrm{Hg}(\mathrm{OH}) 2 \\
=\mathrm{HgCl}-2+2 \mathrm{H} 2 \mathrm{O}\end{array}$ & 20.5338 & 21.794 & 21.79 & & & \\
\hline НgCIOH & $\begin{array}{l}\mathrm{Cl}-+\mathrm{H}++\mathrm{Hg}(\mathrm{OH}) 2= \\
\mathrm{HgClOH}+\mathrm{H} 2 \mathrm{O}\end{array}$ & 9.317 & 10.444 & 10.444 & & & \\
\hline $\mathrm{HgCO}_{3}$ & $\begin{array}{l}\mathrm{Hg}(\mathrm{OH}) 2+2 \mathrm{H}++\mathrm{CO} 3- \\
2=\mathrm{HgCO} 3+2 \mathrm{H} 2 \mathrm{O}\end{array}$ & & 18.272 & 18.29 & & & \\
\hline $\mathrm{HgF}^{+}$ & $\begin{array}{l}\mathrm{F}-+2 \mathrm{H}++\mathrm{Hg}(\mathrm{OH}) 2= \\
\mathrm{HgF}++2 \mathrm{H} 2 \mathrm{O}\end{array}$ & 8.0848 & 7.763 & 7.76 & & & \\
\hline $\mathrm{HgHCO}_{3}^{+}$ & $\begin{array}{l}\mathrm{Hg}(\mathrm{OH}) 2+3 \mathrm{H}++\mathrm{CO} 3- \\
2=\mathrm{HgHCO} 3++2 \mathrm{H} 2 \mathrm{O}\end{array}$ & & 22.542 & & & & \\
\hline $\mathrm{HgHS}_{2}^{-}$ & $\begin{array}{l}\mathrm{Hg}(\mathrm{OH}) 2+\mathrm{H}++2 \mathrm{HS}- \\
=\mathrm{HgHS} 2-+2 \mathrm{H} 2 \mathrm{O}\end{array}$ & & $38.122 / 38.642 *$ & $38.09 / 38.642 *$ & & & \\
\hline $\mathbf{H g R}^{+} \sim_{\text {fulvic acid }}$ & $\mathrm{Hg} 2++\mathrm{R} \sim=\mathrm{HgR}+\sim$ & & & & & '(3.51) & 5.1 \\
\hline
\end{tabular}


Table S4. Used equilibrium constants (log K values) of aqueous Hg species and complexes - Continued.

AQUEOUS COMPLEXES

\begin{tabular}{|c|c|c|c|c|c|c|c|}
\hline \multirow[t]{2}{*}{ Name } & \multirow[t]{2}{*}{ Reaction } & \multirow{2}{*}{$\begin{array}{r}\text { PHREEQC } \\
\text { minteq.dat } \\
\log K \\
\end{array}$} & \multirow{2}{*}{$\begin{array}{c}\text { PHREEQC } \\
\text { minteq.v4.dat } \\
\log \mathrm{K}\end{array}$} & \multicolumn{2}{|c|}{$\begin{array}{l}\text { Vis ual Minteq the rmo.vdb } \\
\text { and shmgene ric } 14 . v d b\end{array}$} & \multicolumn{2}{|c|}{$\begin{array}{c}\text { WHAM } \\
\text { default.db7 }\end{array}$} \\
\hline & & & & $\log K$ & $\Delta \mathbf{L K}_{2}$ & $\log K$ & $\Delta \mathbf{L K}_{2}$ \\
\hline$\overline{H g O H R} \sim$ fulvic acid & $\mathrm{HgOH}++\mathrm{R} \sim=\mathrm{HgR}+\sim$ & & & & & $(3.51)$ & 5.1 \\
\hline $\mathrm{HgR}^{+} \sim_{\text {humic acid }}$ & $\mathrm{Hg} 2++\mathrm{R} \sim=\mathrm{HgR}+\sim$ & & & & & (3.51) & 5.1 \\
\hline HgOHR humic acid & $\mathrm{HgOH}++\mathrm{R} \sim=\mathrm{HgR}+\sim$ & & & & & (3.51) & 5.1 \\
\hline $\mathrm{HgNH}_{3}{ }^{2+}$ & $\begin{array}{l}\mathrm{H}++\mathrm{Hg}(\mathrm{OH}) 2+\mathrm{NH} 4+ \\
=\mathrm{HgNH} 3+2+2 \mathrm{H} 2 \mathrm{O}\end{array}$ & 5.6139 & 5.75 & & & & \\
\hline $\mathrm{HgN}_{3}^{+}$ & $\begin{array}{l}2 \mathrm{H}++\mathrm{Hg}(\mathrm{OH}) 2+\mathrm{N} 3- \\
=\mathrm{HgN} 3++2 \mathrm{H} 2 \mathrm{O}\end{array}$ & & & 13.96 & & & \\
\hline $\mathrm{HgNO}_{2}^{+}$ & $\begin{array}{l}2 \mathrm{H}++\mathrm{Hg}(\mathrm{OH}) 2+\mathrm{NO} 2- \\
=\mathrm{HgNO} 2++2 \mathrm{H} 2 \mathrm{O}\end{array}$ & & & 12.1 & & & \\
\hline $\mathrm{HgNO}_{3}^{+}$ & $\begin{array}{l}2 \mathrm{H}++\mathrm{Hg}(\mathrm{OH}) 2+\mathrm{NO} 3- \\
=\mathrm{HgNO} 3++2 \mathrm{H} 2 \mathrm{O}\end{array}$ & 6.4503 & 5.7613 & 5.7613 & & & \\
\hline $\mathrm{HgOH}^{+}$ & $\begin{array}{l}\mathrm{H}++\mathrm{Hg}(\mathrm{OH}) 2= \\
\mathrm{HgOH}++\mathrm{H} 2 \mathrm{O}\end{array}$ & 2.6974 & 2.797 & 2.767 & & & \\
\hline $\mathrm{HgOH}^{+}$ & $\mathrm{Hg} 2++\mathrm{OH}-=\mathrm{HgOH}^{+}$ & & & & & 10.6 & \\
\hline $\mathrm{HgOH}_{2}$ & $\mathrm{Hg} 2++2 \mathrm{OH}-=\mathrm{HgOH} 2$ & & & & & 21.83 & \\
\hline $\mathrm{HgOH}_{3}^{-}$ & $\mathrm{Hg} 2++3 \mathrm{OH}-=\mathrm{HgOH} 3-$ & & & & & 20.9 & \\
\hline $\mathrm{HgOHCO}_{3}^{-}$ & $\begin{array}{l}\mathrm{Hg}(\mathrm{OH}) 2+\mathrm{CO} 3-2+\mathrm{H}+ \\
=\mathrm{HgOHCO} 3-+\mathrm{H} 2 \mathrm{O}\end{array}$ & & & 11.36 & & & \\
\hline $\mathrm{HgOHI}$ & $\begin{array}{l}1 \mathrm{H}++\mathrm{Hg}(\mathrm{OH}) 2+\mathrm{I}-= \\
\mathrm{HgOHI}+\mathrm{H} 2 \mathrm{O}\end{array}$ & & & 9.41 & & & \\
\hline $\mathrm{HgOHgSH}$ & $\begin{array}{l}\mathrm{Hg}(\mathrm{OH}) 2+\mathrm{HS}-+\mathrm{H}^{+}= \\
\mathrm{HOHgSH}+\mathrm{H} 2 \mathrm{O}\end{array}$ & & $22.447^{\#}$ & & & & \\
\hline
\end{tabular}


Table S4. Used equilibrium constants (log K values) of aqueous Hg species and complexes - Continued.

AQUEOUS COMPLEXES

\begin{tabular}{|c|c|c|c|c|c|c|c|}
\hline \multirow[t]{2}{*}{ Name } & \multirow[t]{2}{*}{ Reaction } & \multirow{2}{*}{$\begin{array}{c}\text { PHREEQC } \\
\text { minteq.dat } \\
\log \mathrm{K}\end{array}$} & \multirow{2}{*}{$\begin{array}{c}\text { PHREEQC } \\
\text { minteq.v4.dat } \\
\log \mathrm{K}\end{array}$} & \multicolumn{2}{|c|}{$\begin{array}{l}\text { Visual Minteq thermo.vdb } \\
\text { and shmgeneric 14.vdb }\end{array}$} & \multicolumn{2}{|c|}{$\begin{array}{c}\text { WHAM } \\
\text { default.db7 }\end{array}$} \\
\hline & & & & $\log K$ & $\Delta \mathbf{L K}_{2}$ & $\log K$ & $\Delta \mathbf{L K}_{2}$ \\
\hline HgOHSCN & $\begin{array}{l}\mathrm{Hg}(\mathrm{OH}) 2+\mathrm{SCN}-1+ \\
\mathrm{H}+=\mathrm{HgOHSCN}+ \\
\mathrm{H} 2 \mathrm{O}\end{array}$ & & & 12.61 & & & \\
\hline $\mathrm{HgSR}^{+}$ & $\begin{array}{l}\text { RS- }+\mathrm{Hg}(\mathrm{OH}) 2+2 \mathrm{H}+ \\
=\mathrm{HgSR}++2 \mathrm{H} 2 \mathrm{O}\end{array}$ & & $26.145^{\circ}$ & & & & \\
\hline $\mathrm{HgSR}^{+}$ & $\mathrm{Hg} 2++\mathrm{RS}-=\mathrm{HgSR}+$ & & & & & $20^{\circ}$ & \\
\hline $\mathrm{HgS} \mathrm{S}_{2}{ }^{2-}$ & $\begin{array}{l}2 \mathrm{HS}-+\mathrm{Hg}(\mathrm{OH}) 2= \\
\mathrm{HgS2} 2-2+2 \mathrm{H} 2 \mathrm{O}\end{array}$ & 31.2398 & $29.414 / 29.342^{*}$ & 29.38 & & & \\
\hline $\mathrm{HgSO}_{4}$ & $\begin{array}{l}2 \mathrm{H}++\mathrm{Hg}(\mathrm{OH}) 2+\mathrm{SO} 4- \\
2=\mathrm{HgSO} 4+2 \mathrm{H} 2 \mathrm{O}\end{array}$ & 7.4911 & 8.612 & 8.63 & & & \\
\hline $\mathrm{HgSO}_{4}$ & $\mathrm{Hg} 2++\mathrm{SO} 4-2-=\mathrm{HgSO} 4$ & & & & & 2 & \\
\hline RSH & RS- $+\mathrm{H}^{+}$ & & $-10^{\circ}$ & $-10^{\circ}$ & & $-10^{\circ}$ & \\
\hline
\end{tabular}

values in itialics were updated in the database in scenario 5 to 8

values in brackets are intrinsic constants for $\mathrm{Hg}$ binding in the Stockholm Humic Model (Visual MINTEQ) or the Humic Ion Binding Model VII (WHAM), respectively.

* $\log \mathrm{K}$ from Drott et al. 2013

\# $\log$ K from Dyrssen \& Wedborg (1991) as published in Skyllberg (2008). The lower of the two published constants was chosen according to Drott et al. (2013)

${ }^{\circ} \log \mathrm{K}$ from Skyllberg (2008)

All $\log \mathrm{K}$ conversions necessary to fit formats of respective databases were performed using thermodynamic data from Wagman et al. (1982).

Only complexes that can form from measured solution constituents (Tables S4-S6) are shown in this table.

Table S5. Analytical details of $\mathrm{Hg}$ measurements.

\begin{tabular}{cccc}
$\begin{array}{c}\text { sample volume } \\
\text { (single } \\
\text { measurement) }\end{array}$ & $\begin{array}{c}\text { purge } \\
\text { rate }\end{array}$ & $\begin{array}{c}\text { purge } \\
\text { time* }\end{array}$ & $\begin{array}{c}\text { calibration } \\
\text { range }\end{array}$ \\
\hline $\mathrm{mL}$ & $\mathrm{L} \mathrm{min}^{-1}$ & $\min$ & pmol \\
\hline $0.05-10$ & 1.1 & $1-2$ & $25-175$ \\
\hline
\end{tabular}

\# the sample volume depended on the Hg concentration of the sample

* the purging time depended on the time until $\mathrm{Hg}$ in the purge air (pumped in a closed circuit) reached a concentration plateau 
Table S6. Limits of quantification, applied standard reference materials, and recovery rates for different analytes.

\begin{tabular}{|c|c|c|c|c|c|c|c|c|}
\hline Analyte & Unit & $\begin{array}{l}\text { Analytical } \\
\text { Instrument }\end{array}$ & $\begin{array}{c}\text { Limit of } \\
\text { Quantification } \\
\text { (LOQ)* }\end{array}$ & $\begin{array}{c}\text { Standard } \\
\text { Reference } \\
\text { Material (SRM) } \\
\end{array}$ & $\begin{array}{r}\text { Certi } \\
\text { Concent }\end{array}$ & $\begin{array}{l}\text { ied } \\
\text { ration }\end{array}$ & $\begin{array}{c}\text { Obtained } \\
\text { Concentration }\end{array}$ & $\begin{array}{c}\text { Recovery } \\
\text { Rate }\end{array}$ \\
\hline $\mathrm{Hg}^{\#}$ & $\begin{array}{l}\text { absolute } \\
\text { Hg in } \\
\text { sample } \\
\text { (pmol) }\end{array}$ & CV-AAS & 3.9 & & & & & \\
\hline $\mathrm{Hg}^{\#}$ & $\begin{array}{l}\mathrm{nmol} \cdot \mathrm{L}^{-1} \\
(20 \mathrm{~mL} \\
\text { sample } \\
\text { volume })\end{array}$ & & 0.20 & $\begin{array}{c}\text { "Trace Metals" } \\
\text { RTC } 1 \text {-WP } \\
\text { (0.29 mL sample } \\
\text { volume })\end{array}$ & $389 \pm$ & 20 & $393 \pm 7.5$ & $101.0 \%$ \\
\hline DOC & $\mu \mathrm{mol} \cdot \mathrm{L}^{-1}$ & $\begin{array}{l}\text { TIC/TOC } \\
\text { Analyzer }\end{array}$ & 42 & $\begin{array}{l}\text { "natural water } \\
\text { from Lake } \\
\text { Superior" EC } \\
\text { ION-915 }\end{array}$ & $114 \pm$ & 34 & $110 \pm 12$ & $96.8 \%$ \\
\hline $\mathrm{Cl}$ & $\mu \mathrm{mol} \cdot \mathrm{L}^{-1}$ & IC & 13.9 & $"$ & $40.1 \pm$ & 6.0 & $40.2 \pm 2.5$ & $100.3 \%$ \\
\hline $\mathrm{F}^{-}$ & $\mu \mathrm{mol} \cdot \mathrm{L}^{-1}$ & IC & 0.7 & $"$ & 2.5 & & 2.1 & $83.9 \%$ \\
\hline $\mathrm{NO}_{3}^{-}$ & $\mu \mathrm{mol} \cdot \mathrm{L}^{-1}$ & IC & 0.3 & $"$ & $1.6 \pm$ & 0.2 & $1.4 \pm 0.1$ & $90.1 \%$ \\
\hline $\mathrm{SO}_{4}{ }^{2-}$ & $\mu \mathrm{mol} \cdot \mathrm{L}^{-1}$ & IC & 7.8 & & $3.4 \pm$ & 0.4 & $3.4 \pm 0.3$ & $100.7 \%$ \\
\hline $\mathrm{Br}^{-}$ & $\mu \mathrm{mol} \cdot \mathrm{L}^{-1}$ & IC & 0.3 & $"$ & & & $0.01 \pm 0.00$ & \\
\hline $\mathrm{Ca}$ & $\mathrm{mmol} \cdot \mathrm{L}^{-1}$ & ICP-OES & 0.08 & $\begin{array}{c}\text { "River Water" } \\
\text { NRC SLRS-5 }\end{array}$ & $0.26 \pm$ & 0.01 & $0.27 \pm 0.00$ & $102.6 \%$ \\
\hline $\mathrm{Na}$ & $\mu \mathrm{mol} \cdot \mathrm{L}^{-1}$ & ICP-OES & 140 & $"$ & $234.0 \pm$ & 4.3 & $237.3 \pm 0.6$ & $101.4 \%$ \\
\hline $\mathrm{K}$ & $\mu \mathrm{mol} \cdot \mathrm{L}^{-1}$ & ICP-OES & 22.2 & $"$ & $21.5 \pm$ & 1.0 & $19.1 \pm 1.0$ & $89.1 \%$ \\
\hline $\mathrm{Mg}$ & $\mu \mathrm{mol} \cdot \mathrm{L}^{-1}$ & ICP-OES & 36.6 & $"$ & $118.5 \pm$ & 8.9 & $123.3 \pm 0.4$ & $104.1 \%$ \\
\hline $\mathrm{Mn}$ & $\mathrm{nmol} \cdot \mathrm{L}^{-1}$ & ICP-OES & 255 & $"$ & $78.8 \pm$ & 3.3 & $79.7 \pm 3.1$ & $101.1 \%$ \\
\hline $\mathrm{Si}$ & $\mu \mathrm{mol} \cdot \mathrm{L}^{-1}$ & ICP-OES & 5 & $"$ & $42.0 \pm$ & 4.8 & $44.6 \pm 0.2$ & $106.1 \%$ \\
\hline
\end{tabular}

* Calculated from the respective calibration according to DIN 32645 or Neitzel (2002).

\# Because the CV-AAS used for $\mathrm{Hg}$ determination was calibrated with absolute $\mathrm{Hg}$ amounts, analytical limits are also absolute $\mathrm{Hg}$ quantities and can only be transformed to concentrations by dividing them through the respective sample volume. This is done in the second row for the maximum sample volume used for $\mathrm{Hg}$ determination $(20 \mathrm{~mL})$ in this study. Because the LOQ was calcutated from the calibration of the CV-AAS, it is identical for all directly measured $\mathrm{Hg}$ species $\left(\mathrm{Hg}_{\text {inorg }}, \mathrm{Hg}(0)\right.$, and $\mathrm{Hg}_{\text {tot }}$ ). 
Table S7. Combined Root Mean Square Errors (RMSEs) and Mean Signed Differences (MSDs) of modeled Hg speciation compared with measured $\mathrm{Hg}$ species $(\log \mathrm{mol}\urcorner \mathrm{L}-1)$ in all samples from all sites.

\begin{tabular}{|c|c|c|c|c|c|c|c|c|c|c|c|c|}
\hline & & & & oot Mean Sq & ared Er & or (RMS & & & Mean Signe & Differer & e (MSD) & \\
\hline & $\begin{array}{l}\text { Modeling } \\
\text { Scenario }\end{array}$ & Applied Code & $\begin{array}{c}\text { DOM } \\
\text { bound } \\
\text { Hg }\end{array}$ & $\begin{array}{c}\text { tot inorg. } \\
\mathrm{Hg}\end{array}$ & $\begin{array}{l}\text { inorg. } \\
\mathrm{Hg}(\mathrm{II})\end{array}$ & $\begin{array}{c}\text { tot } \\
\mathbf{H g}(\mathrm{II})\end{array}$ & $\mathbf{H g}(\mathbf{0})$ & $\begin{array}{c}\text { DOM } \\
\text { bound } \\
\text { Hg }\end{array}$ & $\begin{array}{c}\text { tot inorg. } \\
\mathrm{Hg}\end{array}$ & $\begin{array}{l}\text { inorg. } \\
\mathrm{Hg}(\mathrm{II})\end{array}$ & $\begin{array}{c}\text { tot } \\
\mathrm{Hg}(\mathrm{II})\end{array}$ & $\mathbf{H g}(\mathbf{0})$ \\
\hline & & V-MINTEQ & & & & 0.18 & 2.22 & & & & -0.03 & -1.22 \\
\hline @ & 1 "no DOM" & PHREEQC (minteq.dat) & & & & 1.04 & 1.55 & & & & -0.58 & 0.23 \\
\hline & & PHREEQC (minteq.v4.dat) & & & & 0.26 & 2.26 & & & & -0.05 & -1.28 \\
\hline & & V-MINTEQ SHM & 1.38 & 2.59 & 2.41 & 0.06 & 3.90 & 1.09 & -1.94 & -1.92 & 0.04 & -3.24 \\
\hline & $2 " 100 \% F^{\prime \prime}$ & V-MINTEQ ND & 1.58 & 5.20 & 5.82 & 0.04 & 7.26 & 1.34 & -4.26 & -5.23 & 0.03 & -6.47 \\
\hline 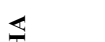 & & WHAM & 1.34 & 2.65 & & & & 1.11 & -1.96 & & & \\
\hline$\sum_{\pi}^{ \pm}$ & & V-MINTEQ SHM & 1.35 & 2.25 & 2.11 & 0.05 & 3.60 & 1.06 & -1.66 & -1.63 & 0.04 & -2.95 \\
\hline$\frac{5}{\pi}$ & 3 "default DOM" & V-MINTEQ ND & 1.56 & 4.95 & 5.55 & 0.04 & 7.00 & 1.33 & -4.01 & -4.94 & 0.03 & -6.17 \\
\hline 递 & & V-MINTEQ SHM & 0.93 & 0.27 & 0.36 & 0.11 & 2.33 & 0.50 & -0.09 & -0.13 & -0.01 & -1.44 \\
\hline & 4 "Malcolm" & V-MINTEQ ND & 1.32 & 3.11 & 3.76 & 0.05 & 5.47 & 1.17 & -1.99 & -2.90 & 0.05 & -5.18 \\
\hline & & WHАM & 0.89 & 0.49 & & & & 0.59 & -0.17 & & & \\
\hline & & PHREEQC (minteq.v4.dat) & 0.83 & 4.17 & 4.17 & 0.06 & 3.83 & 0.52 & -1.57 & -1.58 & 0.01 & -2.18 \\
\hline & 5 "0.15\% thiols" & V-MINTEQ & 0.82 & 4.87 & 4.86 & 0.61 & 5.00 & 0.51 & -1.49 & -1.48 & -0.27 & -2.86 \\
\hline$\frac{n}{\varrho}$ & & WHAM & 0.83 & 4.39 & & & & 0.52 & -1.64 & & & \\
\hline$\tilde{\omega}$ & & PHREEQC (minteq.v4.dat) & 0.64 & 0.15 & 0.26 & 0.13 & 2.31 & 0.03 & 0.04 & 0.02 & -0.01 & -1.34 \\
\hline$\sum$ & $\begin{array}{l}\text { o optimized } \\
\text { thiols" }\end{array}$ & V-MINTEQ & 0.64 & 0.15 & 0.19 & 0.61 & 2.36 & 0.02 & 0.04 & 0.05 & -0.28 & -1.45 \\
\hline 乌 & & WHAM & 0.64 & 0.15 & & & & 0.02 & 0.04 & & & \\
\hline$\approx \mathrm{E} \ll$ & 7 "Malcolm + & V-MINTEQ SHM & 0.65 & 0.15 & 0.22 & 0.62 & 2.27 & 0.07 & 0.03 & 0.03 & -0.29 & -1.29 \\
\hline$\sum_{0}^{\pi} \overline{ \pm}$ & optimized thiols" & WHAM & 0.64 & 0.14 & & & & 0.07 & 0.03 & & & \\
\hline อ & 8 "best fit" & WHAM & 0.59 & 0.13 & & & & 0.03 & 0.04 & & & \\
\hline
\end{tabular}


Table S8. Linear correlation analyses between the measured and the modeled concentrations $(\log (\mathrm{M}))$ )of total inorganic Hg.

\begin{tabular}{|c|c|c|c|c|c|c|c|}
\hline & Scenario & Name & Intercept & Slope & $\mathbf{R}^{2}$ & adj. $\mathbf{R}^{2}$ & p-value \\
\hline \multirow{8}{*}{ 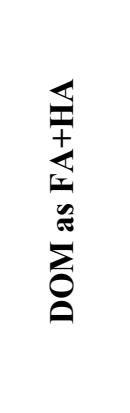 } & \multirow{3}{*}{2} & V-MINTEQ SHM "100\% FA" & 10.83 & 2.86 & 0.72 & 0.70 & $1.92 \mathrm{E}-07$ \\
\hline & & V-MINTEQ ND "100\% FA" & 21.20 & 4.75 & 0.76 & 0.74 & $2.54 \mathrm{E}-06$ \\
\hline & & WHAM "100\% FA" & 10.88 & 2.87 & 0.69 & 0.68 & $5.12 \mathrm{E}-07$ \\
\hline & \multirow{2}{*}{3} & V-MINTEQ SHM "default DOM" & 9.47 & 2.62 & 0.72 & 0.70 & $1.86 \mathrm{E}-07$ \\
\hline & & V-MINTEQ ND "default DOM" & 20.59 & 4.62 & 0.75 & 0.73 & $3.57 \mathrm{E}-06$ \\
\hline & \multirow{3}{*}{4} & V-MINTEQ SHM "Malcolm" & 0.56 & 1.10 & 0.89 & 0.89 & $3.31 \mathrm{E}-12$ \\
\hline & & V-MINTEQ ND "Malcolm" & 10.03 & 2.75 & 0.56 & 0.50 & $1.97 \mathrm{E}-02$ \\
\hline & & WHAM "Malcolm" & 1.15 & 1.19 & 0.76 & 0.75 & $2.35 \mathrm{E}-08$ \\
\hline \multirow{6}{*}{ 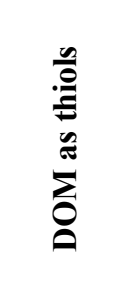 } & \multirow{3}{*}{5} & PHREEQC mit Hg0 "0.15\% thiols" (minteq.v4.dat) & 19.50 & 4.08 & 0.41 & 0.38 & $8.22 \mathrm{E}-04$ \\
\hline & & V-MINTEQ "0.15\% thiols" & 20.62 & 4.22 & 0.32 & 0.28 & $4.28 \mathrm{E}-03$ \\
\hline & & WHAM "0.15\% thiols" & 20.29 & 4.20 & 0.39 & 0.36 & $1.14 \mathrm{E}-03$ \\
\hline & \multirow{3}{*}{6} & PHREEQC (minteq.v4.dat) "optimized thiols" & -0.04 & 0.99 & 0.95 & 0.95 & $3.33 \mathrm{E}-16$ \\
\hline & & V-MINTEQ "optimized thiols" & -0.04 & 0.99 & 0.95 & 0.95 & $3.33 \mathrm{E}-16$ \\
\hline & & WHAM "optimized thiols" & -0.04 & 0.99 & 0.95 & 0.95 & $3.33 \mathrm{E}-16$ \\
\hline \multirow{3}{*}{ 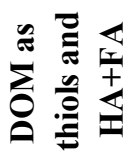 } & \multirow{2}{*}{7} & WHAM "Malcolm + optimized thiols" & 0.07 & 1.01 & 0.96 & 0.96 & $1.11 \mathrm{E}-16$ \\
\hline & & V-Minteq SHM "Malcolm + optimized thiols" & -0.01 & 0.99 & 0.95 & 0.95 & $5.55 \mathrm{E}-16$ \\
\hline & 8 & WHAM "best fit" & -0.12 & 0.98 & 0.97 & 0.96 & $1.00 \mathrm{E}-17$ \\
\hline
\end{tabular}


Table S9. Linear correlation analyses between the measured and the modeled concentrations $(\log (\mathrm{M}))$ of DOM-bound Hg.

\begin{tabular}{|c|c|c|c|c|c|c|c|}
\hline & Scenario & Name & Intercept & Slope & $\mathbf{R}^{2}$ & adj. $R^{2}$ & p-value \\
\hline \multirow{8}{*}{ 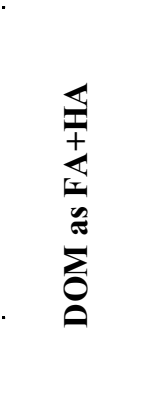 } & \multirow{3}{*}{2} & V-MINTEQ SHM "100\% FA" & -7.04 & -0.01 & 0.00 & -0.05 & $9.28 \mathrm{E}-01$ \\
\hline & & V-MINTEQ ND "100\% FA" & -6.15 & 0.08 & 0.02 & -0.05 & 6.14E-01 \\
\hline & & WHAM "100\% FA" & -6.04 & 0.11 & 0.05 & 0.01 & 2.89E-01 \\
\hline & \multirow{2}{*}{3} & V-MINTEQ SHM "default DOM" & -6.97 & 0.00 & 0.00 & -0.05 & $9.78 \mathrm{E}-01$ \\
\hline & & V-MINTEQ ND "default DOM" & -6.16 & 0.08 & 0.02 & -0.04 & 5.95E-01 \\
\hline & \multirow{3}{*}{4} & V-MINTEQ SHM "Malcolm" & -7.09 & 0.06 & 0.02 & -0.03 & $5.68 \mathrm{E}-01$ \\
\hline & & V-MINTEQ ND "Malcolm" & -9.49 & -0.26 & 0.26 & 0.16 & $1.59 \mathrm{E}-01$ \\
\hline & & WHAM "Malcolm" & -5.96 & 0.19 & 0.21 & 0.17 & 2.51E-02 \\
\hline \multirow{6}{*}{ 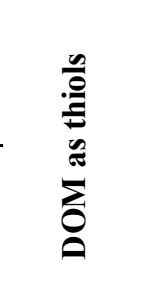 } & \multirow{3}{*}{5} & PHREEQC mit $\mathrm{Hg} 0$ "0.15\% thiols" (minteq.v4.dat) & -5.95 & 0.20 & 0.33 & 0.30 & 3.05E-03 \\
\hline & & V-MINTEQ " $0.15 \%$ thiols" & -6.02 & 0.19 & 0.33 & 0.30 & $3.58 \mathrm{E}-03$ \\
\hline & & WHAM "0.15\% thiols" & -5.95 & 0.20 & 0.33 & 0.30 & 3.05E-03 \\
\hline & \multirow{3}{*}{6} & PHREEQC (minteq.v4.dat) "optimized thiols" & -6.27 & 0.22 & 0.31 & 0.28 & 4.76E-03 \\
\hline & & V-MINTEQ "optimized thiols" & -6.27 & 0.22 & 0.31 & 0.28 & $4.76 \mathrm{E}-03$ \\
\hline & & WHAM "optimized thiols" & -6.27 & 0.22 & 0.31 & 0.28 & 4.76E-03 \\
\hline \multirow{3}{*}{ 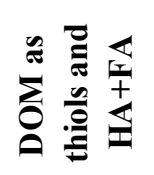 } & \multirow{2}{*}{7} & WHAM "Malcolm + optimized thiols" & -6.40 & 0.20 & 0.27 & 0.24 & 8.73E-03 \\
\hline & & V-Minteq SHM "Malcolm + optimized thiols" & -6.20 & 0.22 & 0.31 & 0.28 & $4.36 \mathrm{E}-03$ \\
\hline & 8 & WHAM "best fit" & -5.83 & 0.27 & 0.45 & 0.42 & 3.37E-04 \\
\hline
\end{tabular}




\section{REFERENCES}

DIN Deutsches Institut für Normung e. V., DIN German Institute for Standardization, 2008. DIN 32645 (2008-11-00) Chemical analysis - Decision limit, detection limit and determination limit under repeatability conditions - Terms, methods, evaluation (Nachweis-, Erfassungs- und Bestimmungsgrenze unter Wiederholbedingungen - Begriffe, Verfahren, Auswertung).

Drott, A., Björn, E., Bouchet, S., Skyllberg, U., 2013. Refining Thermodynamic Constants for Mercury(II)-Sulfides in Equilibrium with Metacinnabar at Sub-Micromolar Aqueous Sulfide Concentrations. Environ. Sci. Technol. 47, 4197-4203. doi:10.1021/es304824n

Dyrssen, D., Wedborg, M., 1991. The sulphur-mercury(II) system in natural waters. Water. Air. Soil Pollut. 56, 507-519. doi:10.1007/BF00342295

EN, European Committee for Standardization, 2007. Method 1483, Water quality- Determination of mercury- Method using atomic absorption spectrometry; German version.

Neitzel, V., 2002. Calibration of Analytical Methods (part 2) non linear calibration functions (Die Kalibration von Analysenverfahren (Teil 2) Nicht lineare Kalibrationsfunktionen). Chem. Labor Biotech. 53.

Skyllberg, U., 2008. Competition among thiols and inorganic sulfides and polysulfides for $\mathrm{Hg}$ and $\mathrm{MeHg}$ in wetland soils and sediments under suboxic conditions: Illumination of controversies and implications for MeHg net production. J. Geophys. Res. 113. doi:10.1029/2008JG000745

USEPA, Office of Science and Technology, 2002. Method 1631, Revision E: Mercury in Water by Oxidation, Purge and Trap, and Cold Vapor Atomic Fluorescence Spectrometry.

Wagman, D.D., Evans, W.H., Parker, V.B., Schumm, R.H., Halow, I., 1982. The NBS Tables of Chemical Thermodynamic Properties. Selected Values for Inorganic and C1 and C2 Organic Substances in SI Units. 